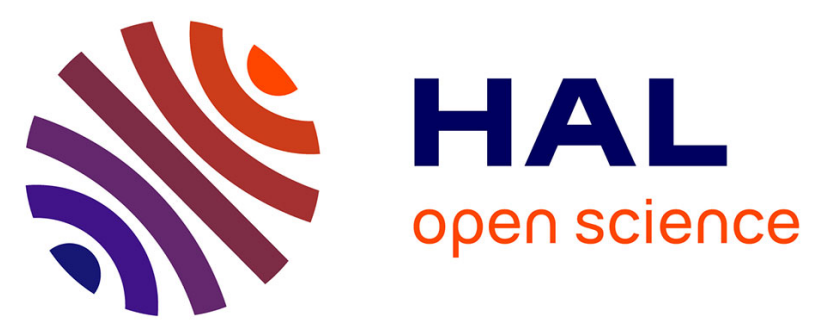

\title{
Generation of multicolor vector Kerr solitons by cross-phase modulation, four-wave mixing, and stimulated Raman scattering
}

Gil Fanjoux, Jeremy Michaud, Michaël Delque, Hervé Maillotte, Thibaut Sylvestre

\section{To cite this version:}

Gil Fanjoux, Jeremy Michaud, Michaël Delque, Hervé Maillotte, Thibaut Sylvestre. Generation of multicolor vector Kerr solitons by cross-phase modulation, four-wave mixing, and stimulated Raman scattering. Optics Letters, 2006, 31 (23), pp.3480-3482. 10.1364/OL.31.003480 . hal-00268719

\section{HAL Id: hal-00268719 https://hal.science/hal-00268719}

Submitted on 29 Aug 2013

HAL is a multi-disciplinary open access archive for the deposit and dissemination of scientific research documents, whether they are published or not. The documents may come from teaching and research institutions in France or abroad, or from public or private research centers.
L'archive ouverte pluridisciplinaire HAL, est destinée au dépôt et à la diffusion de documents scientifiques de niveau recherche, publiés ou non, émanant des établissements d'enseignement et de recherche français ou étrangers, des laboratoires publics ou privés. 


\title{
Generation of multicolor vector Kerr solitons by cross-phase modulation, four-wave mixing, and stimulated Raman scattering
}

\author{
Gil Fanjoux, Jérémy Michaud, Michaël Delqué, Hervé Maillotte, and Thibaut Sylvestre \\ Département d'Optique PM Duffieux, Institut FEMTO-ST, UMR CNRS-Université de Franche-Comté no. 6174, Route \\ de Gray, 25030 Besançon Cedex, France
}

Received June 15, 2006; revised August 28, 2006; accepted September 7, 2006;

posted September 12, 2006 (Doc. ID 71902); published November 9, 2006

\begin{abstract}
We numerically and experimentally show the existence of multicolor vector spatial solitons in a Kerr planar waveguide through the combined effects of cross-phase modulation, four-wave mixing, and stimulated Raman scattering. Mutual spatial guiding of the Raman-Stokes, anti-Stokes, and pump waves is achieved in the high-conversion regime mainly by cross-phase modulation and phase-matched four-wave mixing induced by a power imbalance between Stokes and anti-Stokes components, leading to the generation of a clear-cut sech-shape three-frequency spatial soliton. (c) 2006 Optical Society of America

OCIS codes: $190.0190,190.5530,190.3270,190.5650,190.5890,190.4380$.
\end{abstract}

Among the large family of optical solitons, vector solitary waves are currently the subject of many studies owing to the additional appeal of their multicomponent nature. The existence of such entities relies on a balanced interaction of several field components (different frequencies, polarizations, or modes) in such a way that shape-preserving propagation is allowed. Each field component is mutually guided by the others but could not have a solitonic propagation alone. An example of multicomponent vector solitons is the multicolor soliton (MS). Until now, this soliton has been observed to a great extent in quadratic media. ${ }^{1}$ The coupling processes are fundamentally different than those occurring in Kerr media and are based on the focusinglike phase-front curvature due to the cascading process and the beam narrowing due to the parametric gain. ${ }^{2}$ A multifrequency spatial soliton has also been reported in a Raman gas medium strongly driven near maximum molecular coherence by two lasers slightly detuned from the Raman resonance. ${ }^{3}$ However, in cubic transparent media, the generation of the MS has not yet been fully addressed. ${ }^{4-7}$ From a theoretical viewpoint, it was first predicted that the MS could exist in focusing Kerr media, assuming only cross-phase modulation (XPM) as a coupling process. ${ }^{4}$ Now, if the phase mismatch between the interacting fields is low, or, more interestingly, when powers are sufficient for producing a nonlinear phase change that can counterbalance the linear phase mismatch, coherent coupling by four-wave mixing (FWM) must be considered. By considering self-phase modulation (SPM), XPM, and FWM, Chen and Atai ${ }^{5}$ later showed that the MS involving three-frequency trapped beams can exist below a critical frequency shift for which the three waves are either phase matched or highly phase mismatched. Another recent work has shown that, above a threshold power, FWM leads to the instability of two-color solitons, and they appear as a three-color soliton for which the parametric coupling is exactly balanced by SPM and XPM. $^{6}$

In this Letter, we present numerical and experimental results that show the spontaneous generation of a three-frequency spatial soliton in a Kerr $\mathrm{CS}_{2}$ planar isotropic waveguide when stimulated Raman scattering (SRS) enters into play. Mutual spatial guiding of the Raman-Stokes, anti-Stokes, and pump waves is achieved in the strong-conversion SRS regime mainly by XPM and phase-matched FWM induced by power imbalance, leading to a clear-cut sech-shape three-frequency spatial Raman soliton.

Let us first consider the following three coupled nonlinear Schrödinger scalar equations in the (1 $+1) D$ configuration for the pump $(p)$, the first-order Raman-Stokes (+), and the anti-Stokes (-) waves:

$$
\begin{aligned}
\frac{\partial E_{p}}{\partial z}= & \frac{i}{2 \beta_{p}} \frac{\partial^{2} E_{p}}{\partial x^{2}}+i \gamma_{p}\left[P_{p}+2 P_{+}+2 P_{-}\right] E_{p} \\
& +2 i \gamma_{p} E_{p}^{*} E_{+} E_{-} e^{-i \Delta \beta z}-g_{+} P_{+} E_{p}+g_{-} P_{-} E_{p}
\end{aligned}
$$

$$
\begin{aligned}
\frac{\partial E_{ \pm}}{\partial z}= & \frac{i}{2 \beta_{ \pm}} \frac{\partial^{2} E_{ \pm}}{\partial x^{2}}+i \gamma_{ \pm}\left[P_{ \pm}+2 P_{p}+2 P_{\mp}\right] E_{ \pm} \\
& +i \gamma_{ \pm} E_{\mp}^{*} E_{p}^{2} e^{\mathrm{i} \Delta \beta z} \pm g_{ \pm} P_{p} E_{ \pm},
\end{aligned}
$$

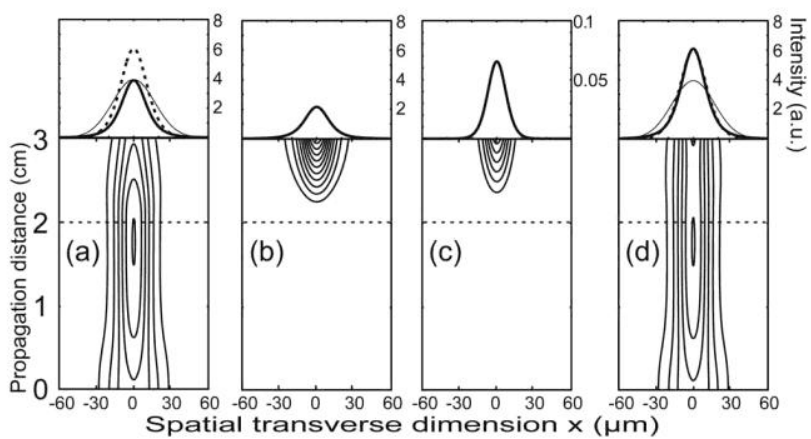

Fig. 1. Numerical simulation of the generation of a threefrequency spatial soliton. Bottom: contour plots of (a) the pump, (b) Stokes, (c) anti-Stokes, and (d) whole beam. Top, transverse beam profiles at the waveguide input (thin solid curve), output (bold solid curve), and after a $2 \mathrm{~cm}$ propagation (dashed curve). The parameters are input width $=40 \mu \mathrm{m}, \mathcal{P}_{\text {sat }}=0.02 \mathrm{~W} / \mathrm{m}, g_{r}=23.2 \mathrm{~cm} / \mathrm{MW} . \mathcal{P}_{p}=1.6 \mathcal{P}_{s}$. 

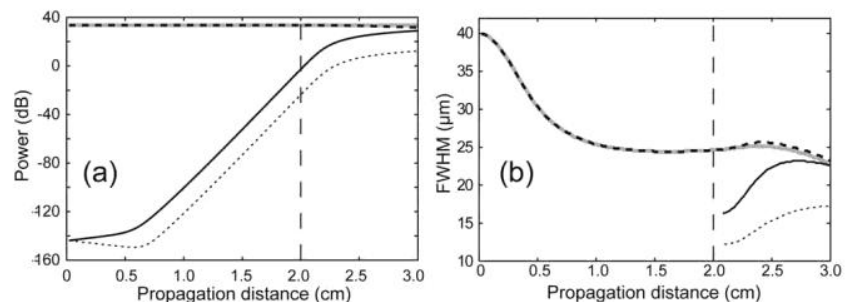

Fig. 2. Evolution along the waveguide of (a) powers and (b) FWHM of the pump (bold dashed curve), Raman-Stokes (bold solid curve), Raman-anti-Stokes (thin dashed curve) components, and total output beam (gray curve).

where $E_{j}(x, z)$ is the amplitude of the field $j(j=p,+$, $-), P_{j}=\left|E_{j}\right|^{2}$ is defined in watts per square meter, $\gamma_{j}$ $=2 \pi n_{2} / \lambda_{j}$ is the nonlinear parameter where $n_{2}\left(n_{2}=\right.$ $+3.5 \times 10^{-18} \mathrm{~m}^{2} / \mathrm{W}$ for $\mathrm{CS}_{2}$ ) is the nonlinear coefficient, and $\lambda_{j}$ is the wavelength of the $j$ th field. $\Delta \beta$ $=2 \beta_{p}-\beta_{+}-\beta_{-}=\beta_{2} \Omega^{2}$ is the linear phase mismatch due to the chromatic dispersion (assuming single-mode and isotropic waveguide) with $\beta_{j}$ as the propagation constant, $\beta_{2}$ as the group-velocity dispersion of the $\mathrm{CS}_{2}$, and $\Omega=\left|\omega_{p}-\omega_{+}\right|$as the Raman shift. $x$ is the free transverse spatial dimension. The Raman coupling process is included in the form of a gain (loss) for the Stokes (anti-Stokes) components. To take the Stokes power saturation due to the Raman cascading into account, the gain for the Stokes component is defined as $g_{+}=g_{r} /\left(1+\mathcal{P} / \mathcal{P}_{\text {sat }}\right)$, while $g_{-}=g_{r}$ with $g_{r}$ as the Raman gain factor, ${ }^{8} \mathcal{P}$ as the power integrated along $x$ (watts per meter) of the Stokes component, and $\mathcal{P}_{\text {sat }}$ as an adjustable parameter. Note that our model does not intrinsically take into account the wave mixing process with the higher Raman orders that should also lead to the generation of a multifrequency soliton.

Numerical simulations of Eqs. (1) and (2) allow one to clearly identify the underlying nonlinear mechanisms for MS generation. Figures 1(a)-1(d) show contour plots of the longitudinal evolution of the spatial profiles of the pump, Stokes, anti-Stokes, and whole beam, respectively. For this simulation, we assumed an input noise level for the Stokes and anti-Stokes components corresponding to the quantum noise. As can be seen in Fig. 1(a), the input Gaussian pump beam first undergoes self-focusing as the input power is greater than the scalar fundamental soliton power $\mathcal{P}_{s}$. Figures 1(b) and 1(c) show the efficient generation of the Stokes and anti-Stokes components beyond $2 \mathrm{~cm}$ propagation and spatially trapped in the pump beam. Thereafter, it is clear that, despite the fact that the three components have varying energies, the total beam exhibits a stationary propagation as shown in Fig. 1(d). This MS generation is confirmed by the transverse beam profiles plotted at the top of Figs. 1(a)-1(d) that show, at the waveguide output, clear reshaping of the input beam into a sech-shape soliton as for both Raman components. We have verified that for a longer propagation distance the MS still propagates in a stable condition. It can be understood as being a composite soliton with variable components mutually trapped through cross-phase modulation. Figures 2(a) and 2(b) show the inte- grated power $\mathcal{P}$ and the full width at half-maximum (FWHM) of the three components in the function of $z$, respectively. We can distinguish two steps of the lowand high-conversion regime separated by a dashed line at $2 \mathrm{~cm}$. The first one is characterized by an exponential growth of the Stokes and anti-Stokes components with a large power imbalance $(\sim 20 \mathrm{~dB})$. Indeed, in spite of a linear phase mismatch between the Stokes and anti-Stokes waves due to chromatic dispersion, FWM occurs because the phase matching is self-induced due to the SRS-induced power imbalance between the two sidebands. ${ }^{9}$ Even if the antiStokes is first attenuated owing to the Raman process, this component subsequently follows the evolution of the Stokes wave through parametric amplification. ${ }^{10}$ This low-conversion regime corresponds to the guiding by XPM of the weak Raman components in the waveguide generated by the pump. ${ }^{11}$ On the other hand, in the strong-conversion regime, the Raman and pump powers become of the same order such that they are mutually guided and form a MS. This is confirmed by Fig. 2(b) because the pump and Stokes components exhibit the same FWHM at the waveguide output, whereas the antiStokes have a lower FWHM due to a lower power. Note that the energy transfer through SRS from the pump to the Raman-Stokes beam annihilates the formation of a higher-order soliton for the pump and leads to the stabilization of the width of the pump Kerr soliton [Fig. 2(b)].

The experimental setup, depicted in Fig. 3, is made up of a $3 \mathrm{~cm}$ long Kerr planar waveguide made up of a thin $15 \mu \mathrm{m}$ liquid $\mathrm{CS}_{2}$ layer sandwiched between two SK5 glass blocks. As a pump laser, we use a frequency-doubled Nd:YAG microchip laser with a $7 \mathrm{kHz}$ repetition rate and $600 \mathrm{ps}$ pulse duration. In $\mathrm{CS}_{2}$, the Raman frequency shift and linewidth are $20 \mathrm{THz}$ and $15 \mathrm{GHz}$, respectively. ${ }^{8}$ To measure the transverse profiles of the pump at $532 \mathrm{~nm}$ and the Stokes at $551 \mathrm{~nm}$ separately, we use a double-grating system (2400 lines $/ \mathrm{mm})$ and a CCD camera, while the whole beam is analyzed by using the zero order of the first grating. Note that the anti-Stokes component at $513 \mathrm{~nm}$ has very low power and has not been analyzed.

Figures 4(a) and 4(b) show the experimental values of the power and the FWHM of the pump and the first-order Raman-Stokes beams at the waveguide output for increasing input power. The numerical predictions (dashed curves) are in good agreement with the experimental measurements. In a similar manner as for Figs. 2(a) and 2(b), two regimes of low-

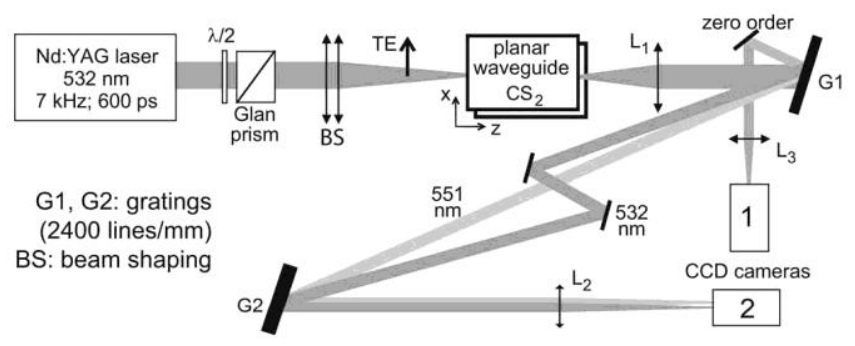

Fig. 3. Experimental setup. 


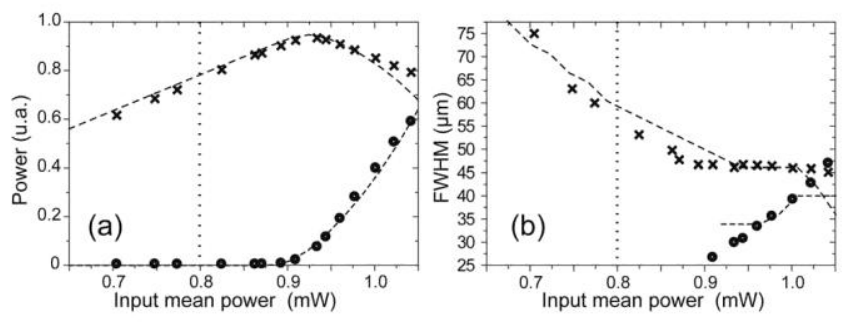

Fig. 4. Experimental (a) output power and (b) FWHM versus input power for the pump $(\times)$ and the Stokes $(\bullet)$ waves. Dashed curves, numerical results. The vertical dotted lines indicate the fundamental soliton mean power $(0.8 \mathrm{~mW})$ for which scalar soliton behavior has been experimentally observed.

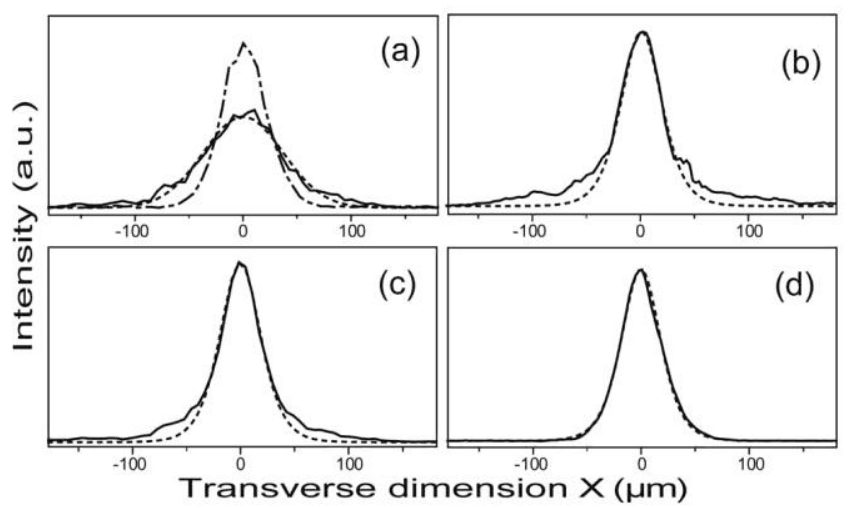

Fig. 5. Black solid curves, experimental intensity profiles of (a) the output beam in linear regime, (b) the pump, (c) the total, and (d) Stokes output beams in the nonlinear regime. The dashed curves correspond to (a) a Gaussian and (b)-(d) to a sech-shape profile, while the dotted-dashed curve in (a) represents the experimental input profile.

and strong-conversion are clearly distinguishable. MS is generated as soon as its components have similar profiles therefore corresponding to a balanced mutual XPM regime. XPM is then the predominant effect in the shape preservation and also in the MS generation. Let us note that the MS does not have an intrinsically stable equilibrium because of the SRSand FWM-induced power transfer (coherent coupling) between the three components of solitons, as observed in Figs. 2(a) and 4(a). However, the whole entity, for which the total energy is conserved, preserves its spatial shape and therefore propagates as a multicolor soliton.

Figures 5(a)-5(d) show the experimental intensity profiles for the pump in the linear and nonlinear regimes, the whole MS and the Stokes beam, respectively. As can be deduced, the total Gaussian input beam exhibits diffraction in the linear regime, while a clean sech-shape MS is generated in the strongconversion regime. The pump [Fig. 5(b)] and Stokes [Fig. 5(d)] components then have mainly the same power and FWHM. Note that the numerical results fit the experimental output profiles for the pump and whole beams very well [Figs. 5(b) and 5(c)] only in the central parts, where beam cleaning due to the spatial soliton effect occurs. More importantly, Fig. 5(d) reveals that the Stokes component of the MS has a clean-cut sech-shape profile and is totally smooth. This is because the Stokes beam is only generated when the pump beam attains a solitonlike regime and does not suffer from noisy nonsolitonic parts. This property can be very useful for a beam cleanup application. $^{12}$

To conclude, we presented numerical and experimental results showing the generation in a Kerr planar waveguide of a multicolor vector Raman soliton composed of three frequencies (pump, RamanStokes, and anti-Stokes beams). Moreover, this work opens new potential applications for simultaneous wavelength conversion and beam cleanup.

This work has been supported by the Ministère Délégué à la Recherche. The authors thank V. Couderc for helpful discussions. G. Fanjoux's e-mail address is gil.fanjoux@univ-fcomte.fr.

\section{References}

1. W. E. Torruellas, L. Torner, Z. Wang, D. J. Hagan, E. W. Van Stryland, G. I. Stegeman, and C. R. Menyuk, Phys. Rev. Lett. 13, 42 (1995).

2. G. Assanto and G. I. Stegeman, Opt. Express 10, 388 (2002).

3. D. D. Yaruv, D. R. Walker, and M. Y. Shverdin, Phys. Rev. A 67, 041803(R) (2003).

4. H. T. Tran, R. A. Sammut, and W. Samir, Opt. Commun. 113, 292 (1994).

5. Y. Chen and J. Atai, Opt. Lett. 19, 1287 (1994).

6. P. B. Lundquist, D. R. Andersen, and Y. S. Kivshar, Phys. Rev. E 57, 3551 (1998).

7. V. Boucher and X. Nguyen-Phu, Appl. Opt. 41, 4390 (2002).

8. R. W. Boyd, in Nonlinear Optics (Academic, 1992), p. 371.

9. S. Coen, D. A. Wardle, and J. D. Harvey, Phys. Rev. Lett. 89, 273901 (2002).

10. Y. R. Shen and N. Bloembergen, Phys. Rev. 137, 1787 (1965).

11. R. De la Fuente and A. Barthélémy, IEEE J. Quantum Electron. 28, 547 (1992).

12. J. Reintjes, R. H. Lehmberg, R. S. F. Chang, M. T. Duilgnan, and G. Calame, J. Opt. Soc. Am. B 3, 1408 (1986). 